at baseline. In the following year assessment subjects were classified as decliners or not according to their worsening nominal performance in GCS or FAQ. Results: Cortisol levels were found to be higher in both cognitively impaired groups $(\mathrm{p}=0.0002)$, and sTNFR1 levels were higher only in dementia group $(p=0.0007)$. In logistic regression analysis adjusted for multiple covariates sTNFR1 levels were associated with prevalent dementia $(\mathrm{OR}=1.0044$; CI:1.002-1.007); instead, cortisol levels were associated (OR:1.084; CI:1.024-1.147) with prevalent cognitive impairment (CIND + dementia), but not with dementia itself, and in a clinical covariate adjusted-model, cortisol levels did not hold statistical association with CIND status. In both groups with cognitive impairment cortisol levels did not correlate with performance in cognitive measures. No biomarker was found to be associated with GCS and FAQ decline according to the employed logistic regression models. CIND status at baseline was the main predictor of functional decline in one year follow-up $(\mathrm{OR}=3.17$; CI:1.191.45). Conclusions: The absence of a graded pattern for cortisol and sTNFR1 serum levels across the three groups categorized according their functional and cognitive status, a lack of correlation between serum levels and measures of cognitive performance, and the absence of associations with the investigated longitudinal outcomes suggest that these biomarkers do not play a lead role in the mechanisms underlying age-associated cognitive and functional impairment.

\section{P2-165 INVESTIGATING THE ROLE OF UBIQUILIN2 IN AGE-RELATED NEURODEGENERATION}

Henry L. Paulson ${ }^{1,2}$, Lisa M. Sharkey ${ }^{2}$, Nate Safren ${ }^{3}$, Amit Pithadia ${ }^{2}$, Svetlana Fischer ${ }^{2}$, Li Zeng ${ }^{2}$,

Mark E. Dulchavsky ${ }^{2}$, Ronak Patel ${ }^{2}$, Gabrielle Lantis ${ }^{2}$, Sami Barmada ${ }^{1,2}$, Magdalena Ivanova ${ }^{2},{ }^{1}$ Michigan Alzheimer's Disease Center, Ann Arbor, MI, USA; ${ }^{2}$ University of Michigan, Ann Arbor, MI, USA; ${ }^{3}$ University of Michigan, Ann Arbor, MI, USA. Contact e-mail: henryp@umich.edu

Background: Brain accumulation of Ubiquilin2 (UBQLN2) is a shared feature of neurodegenerative diseases including Alzheimer's disease, Lewy Body Dementia, Frontotemporal Dementia (FTD) and polyglutamine expansion disorders such as Huntington disease (HD). UBQLN2's involvement in neurodegeneration reflects the fact that it normally helps maintain protein quality control, principally in protein degradation by the proteasome. Rare mutations in $U B Q L N 2$ also directly cause neurodegeneration on the FTD/ALS spectrum. Unfortunately, UBQLN2's normal function in health and its dysfunction in disease remain poorly understood. Methods: To explore UBQLN2-mediated disease we employed protein biochemistry, cell-based studies, longitudinal fluorescence microscopy and mouse models of ALS/FTD and HD. Results: Data from in vitro studies of full length UBQLN2, expression studies in cultured neurons, and transgenic mice expressing wildtype (WT) or mutant (MUT) UBQLN2 support the view that the protein is intrinsically prone to form aggregates and that pathogenic mutations can accelerate this process. Studies of UBQLN2 deleted of functional domains implicate a specific domain in driving aggregation of both wild type and mutant UBQLN2. In transgenic mice expressing MUT-UBQLN2, the protein becomes sequestered in large aggregates within neurons throughout the central nervous system including cortex, hippocampus, cerebellum and spinal cord. Mutant mice also exhibit aberrant TDP43 localization, a common pathological hallmark of ALS/FTD generally and of
UBQLN2-mediated disease specifically. In contrast, WT-UBQLN2 largely remains diffusely distributed in neurons, though some neurons display spherical puncta enriched for UBQLN2. When overexpressed in a mouse model of HD, WT-UBQLN2 nearly abolishes nuclear aggregates of the HD protein, a characteristic disease feature of HD. In the presence of mutant HD protein, UBQLN2 also moves from its normal cytoplasmic location to the nucleus of affected neurons. Conclusions: Our results support a dominant toxic effect of MUT-UBQLN2 driving disease pathogenesis, associated with aggregation of the protein. Our results further support the view that UBQLN2 normally functions in neurons to help maintain protein homeostasis; whether partial loss of this quality control function contributes to disease pathogenesis remains unknown. Accordingly, ongoing studies are focused in part on evaluating the differences between wild type and mutant forms of UBQLN2 in maintaining neuronal protein homeostasis.

\section{P2-166 THE EFFECT OF CHRONIC SLEEP DEPRIVATION ON COGNITION AND BRAIN STRUCTURE IN HEALTHY, MIDDLE-AGED MEN}

Jana Thomas ${ }^{1}$, Sharon Ooms ${ }^{2}$, Jurgen A. H. R. Claassen ${ }^{2}$, Marcel G. M. Olde Rikkert ${ }^{3},{ }^{1}$ Radboudumc, Nijmegen, Netherlands; ${ }^{2}$ Radboud University Medical Center, Nijmegen, Netherlands; ${ }^{3}$ Radboud University Medical Center, Nijmegen, Netherlands. Contact e-mail: jana. thomas@radboudumc.nl

Background:Recent evidence shows a bi-directional relation between poor sleep and the development of Alzheimer's disease (AD). Sleep disorders are more frequent and severe in $\mathrm{AD}$ patients compared to healthy aging people, in turn poor sleep may play a causal role in the pathology of AD by influencing the clearance and production of the amyloid beta protein. Although recent studies have investigated the effect of a night of total sleep deprivation, the effect of chronic sleep deprivation on cognitive decline and $\mathrm{AD}$ has not been researched yet. Objective:To study the relationship between prolonged sleep deprivation, cognitive function and brain structure, in order to determine whether prolonged abnormal sleep behavior has an effect on cognition before subjective cognitive complaints are noticed. Methods: Our study population consists of a group of male volunteers (aged 50 to 60 years), who show irregular sleeping patterns for more than 15 years $(n=20)$. These participants are compared to a group of healthy volunteers with normal sleep, matched in gender, education and age $(n=20)$. All participants will have one night of standard polysomnography (PSG), preceded with two weeks of actigraphy (Actiwatch Philips). Cognition and memory is assessed with a neuropsychological test battery and structural and functional changes of the brain are measured with an MRI scan. Results: The study is currently ongoing and we expect to complete the analysis in June 2017. hypothesize that the chronic sleep deprivation group will show less slow wave sleep, more fragmented sleep and will perform worse on cognitive tests compared to the healthy volunteers. Furthermore, we expect to see subtle biomarkers of neurodegeneration in the sleep deprived group, but not in the healthy control group. Conclusions: This study will investigate the effect of chronic sleep deprivation on both cognition and neurodegeneration even before subjective complaints are reported. Identifying poor sleep as one of the preventable risk factors to develop $\mathrm{AD}$, we will create more awareness about individual sleep behavior and might be able to manage sleep disorders, which will delay the onset of $\mathrm{AD}$ in high risk groups. 